\title{
Sarcoidosis and subsequent cancer risk: a Danish nationwide cohort study
}

To the Editor:

A link between sarcoidosis and malignancy was suggested by in 1972 BRINCKER [1], and subsequently supported by several studies [2-6]. However, the existing studies have different short-comings, and the causation between co-existing sarcoidosis and cancer is still being debated [7].

Initial misdiagnosis of cancer as sarcoidosis may explain a short-term increased cancer risk [6]. In addition, the clinical examinations at routine control visits increase the chance of detecting a malignant tumour (surveillance bias). Chronic inflammation or a dysregulated immune system accompanying sarcoidosis may be the biological mechanism behind an increased long-term cancer risk $[4,8]$. Moreover, sarcoidosis treatment with immunosuppressive agents may increase the risk of later immune-related cancers [9].

We conducted a nationwide cohort study in Denmark (5.5 million residents) and examined the long-term risk of cancer among patients with a hospital-based diagnosis of sarcoidosis. We aimed to estimate potential influence of surveillance bias and immune suppression, thereby adding to the existing literature.

The Danish National Health Service provides free access to hospitals including outpatient clinics, and reimbursement for prescription drugs for all residents.

We used the Danish National Registry of Patients [10] to identify all patients with an inpatient or outpatient diagnosis of sarcoidosis (International Classification of Diseases (ICD) revision 8:135, and ICD-10:D86) in the period 1980 to 2011.

From the Danish National Database of Reimbursed Prescriptions, we ascertained data on use of immunosuppressive agents (glucocorticoid, methotrexate and azathioprine) since 2004 in our cohort, and since 1989 for a sub-group (one-third of the Danish population).

We linked the study cohort to the Danish Cancer Registry [11], and identified incident cancer cases. We excluded patients diagnosed with cancer before the date of sarcoidosis diagnosis.

Follow-up began at the date of first sarcoidosis diagnosis and lasted until first cancer diagnosis, death, emigration or December 2011. We estimated standardised incidence ratios (SIR), a measure of relative risk, comparing cancer incidence observed among patients with sarcoidosis with that expected based on national cancer incidence rates by age, sex and calendar year. We examined the cancer risk according to sex, age, type of sarcoidosis diagnosis (primary or secondary), and type of initial hospital contact (inpatient or outpatient). For periods with available drug data, we repeated long-term analyses for sarcoidosis patients with one or more prescriptions for immunosuppressive agents within 1 year after sarcoidosis diagnosis. We also estimated absolute risks of cancer in patients with sarcoidosis, considering death as a competing risk.

All statistical analyses were conducted using SAS (version 9.2; SAS Institute, Cary, NC, USA). The study was approved by the Danish Data Protection Agency (record number 2013-41-2522).

We identified 12890 patients with a first-time sarcoidosis diagnosis. Median (interquartile range) age was $40(31-54)$ years and $53 \%$ were male. The majority (82\%) had sarcoidosis as their primary diagnosis, and $64 \%$ were diagnosed as outpatients (5496 out of 8552 patients diagnosed during 1995-2011).

Within a median (interquartile range) follow-up of 10 (4-17) years we identified 1303 incident cancers, yielding an overall cancer SIR of 1.3 (95\% CI 1.3-1.4) (table 1). The majority of cancers occurred in patients aged 50-69 years, whereas age did not modify the relative risk. Stratification by primary/secondary diagnosis and inpatient/outpatient diagnosis yielded similar results. In total, 3115 patients had a prescription of immunosuppressive agents, and their median (interquartile range) follow-up was 5 (3-8) years.

The cancer risk was particularly high within the first 3 months, mainly due to an excess occurrence of lung cancer, tonsil cancer and lymphoma. The absolute 3 -month cancer risk was $1.2 \%$ (lung cancer $<0.2 \%$ ). SIRs were also increased for gastrointestinal, urological, haematological and brain cancer during the first 3 months; whereas breast, skin and reproductive system cancers occurred as frequently as expected.

During 3 months to 3 years after sarcoidosis diagnosis, the SIR was moderately increased (mainly based on lung, tonsil, brain and haematological cancers), and the 3 -year absolute cancer risk was $3.2 \%$. In 
TABLE 1 Number of observed cancers and standardised cancer incidence ratios (SIRs) in all sarcoidosis patients and those treated with an immunosuppressant

All sarcoidosis patients ${ }^{\#}$

Sarcoidosis patients treated with

immunosuppressive agents"

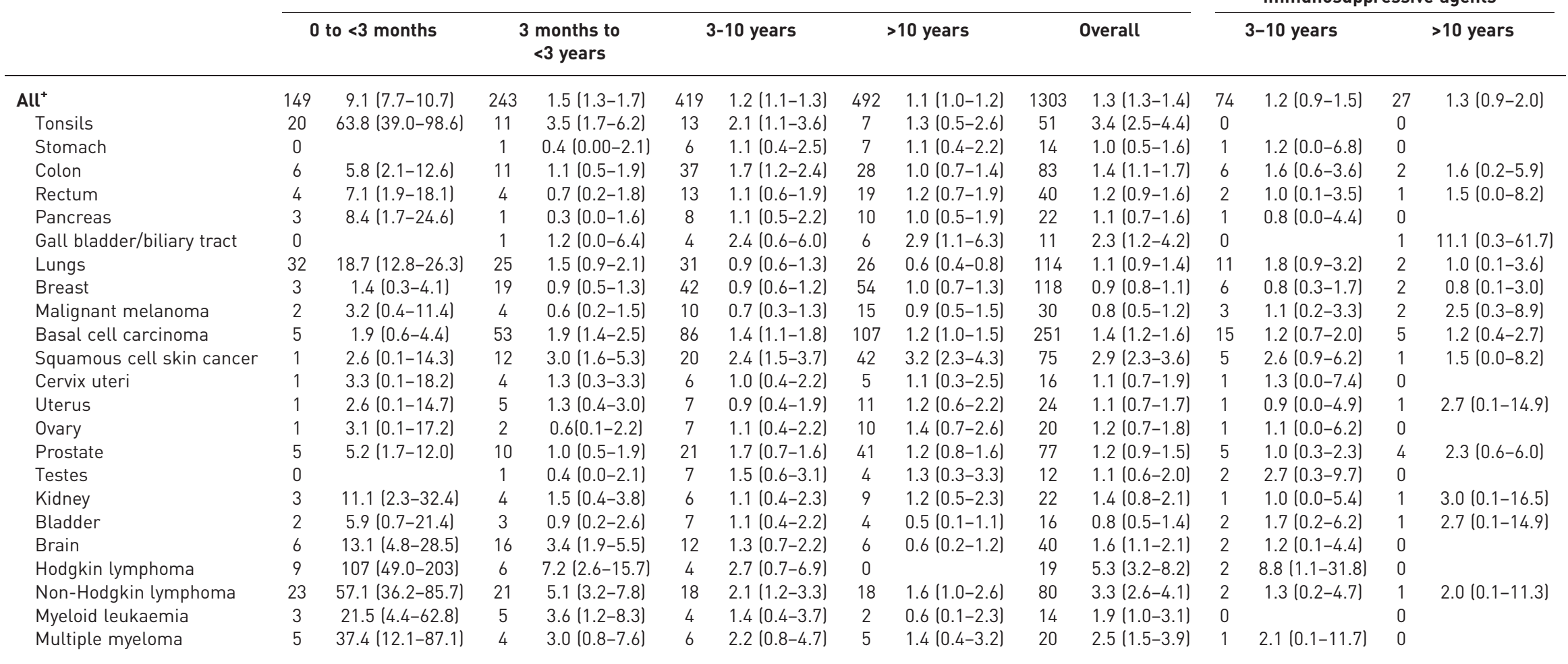

Data are presented as observed number or SIR $(95 \% \mathrm{CI}) .{ }^{\#}: \mathrm{n}=12890 ;{ }^{\Uparrow}: \mathrm{n}=3115 ;{ }^{+}$: the site specific numbers do not add up to the total number because not all cancer sites are presented. 
addition, basal cell carcinoma and squamous cell skin cancer occurred more frequently than expected, while we found no increased risk of gastrointestinal, urological or reproductive system cancers.

Patients with sarcoidosis had $20 \%$ and $10 \%$ increased cancer risk in the periods of 3-10 years and $>10$ years after sarcoidosis diagnosis, respectively. The 10 -year absolute cancer risk was $7.7 \%$. The increased cancer risk beyond 3 years of follow-up was mainly due to a higher than expected occurrence of non-Hodgkin lymphoma and tonsil, colon, gall bladder/biliary tract and non-melanoma skin cancer. The increased risk of gall bladder/ biliary tract cancer, basal cell carcinoma and squamous cell skin cancer persisted even beyond 10 years of follow-up. In contrast, there was no association with lung cancer during 3-10 years of follow-up, and beyond 10 years it was diagnosed less frequently than expected. Restriction to patients with prescriptions of immunosuppressive agents did not further increase the risk, but these estimates had low precision.

In this nationwide study, we confirmed an increased cancer risk shortly after the sarcoidosis diagnosis, but more importantly found a persistent increased risk of several cancers beyond 10 years.

Strengths include the large size, inclusion of outpatient diagnoses and high validity of diagnoses used [10, 11]. Nevertheless, we lacked information on diagnostic work-up, sarcoidosis severity and other clinical characteristics, and potential misclassification may have affected our results.

Our finding of an increased overall risk of cancer among sarcoidosis patients is in accordance with several previous studies using the general population as a comparison [2-6], while one study found no association when comparing sarcoidosis patients with other hospitalised patients [12].

One study argued that misclassification explained the apparent association [6]. We agree that the increased short-term risk of cancer most likely reflects the presence of occult cancer, and that reverse causality, misclassification and increased early surveillance may explain the association for at least the first 3 years after diagnosis, where routine clinical examinations are regularly offered in Denmark.

We confirmed a substantially increased risk of lung cancer during the first 3 months, which was also previously reported [2], but thereafter the risk declined substantially. Regarding the long-term risk of lung cancer, we supported the finding of a decreased risk [12]. A compensatory deficit in the period after the first 3 months conforms to our expectations. However, the finding of a decreased long-term lung cancer risk corresponds poorly with the hypothesis of chronic inflammation playing a key role in the development of cancer in patients with sarcoidosis $[8,13]$.

In agreement with previous studies [2, 4, 5, 14], we also found an increased risk of Hodgkin lymphoma and skin cancer. Moreover, we found a persistent strong association between sarcoidosis and non-Hodgkin lymphoma, which has been less clearly described $[2,4,5,15]$.

Steroid use increases the risk of immune-related cancer [9], including cancers associated with human papillomavirus. We found a substantial increased risk of tonsil cancer, whereas cervical cancer did not occur more often than expected. The results for other human papillomavirus-associated cancers were inconclusive. Moreover, patients using immunosuppressive agents had similar risks as the entire cohort, which, despite lack of statistical precision, may argue against a critical impact of immunosuppressive medicine.

In conclusion, a short-term increased risk of cancer in patients with sarcoidosis was potentially explained by increased surveillance, or initial misinterpretation of cancer as sarcoidosis. In contrast, the increased long-term risk of tonsil cancer, colon cancer and immune-related cancers was notable, and is unlikely to reflect surveillance bias alone.

0 @ERSpublications

Diagnosing work-related asthma decreased asthma-related healthcare utilisation in Quebec and Ontario http://ow.ly/CFrPk

Kirstine Kobberøe Søgaard, Claus Sværke, Reimar Wernich Thomsen and Mette Nørgaard

Dept of Clinical Epidemiology, Institute of Clinical Medicine, Aarhus University Hospital, Aarhus, Denmark.

Correspondence: Kirstine Kobberøe Søgaard, Dept of Clinical Epidemiology, Aarhus University Hospital, Olof Palmes Allé 43-45, 8200 Aarhus N, Denmark. E-mail: kks@clin.au.dk

Received: Jan 142014 | Accepted after revision: June 152014 | First published online: Aug 072014

Support statement: Kirstine Kobberøe Søgaard was supported by a scholarship from Aarhus University (Aarhus, Denmark). This research was supported by the Clinical Epidemiology Research Foundation at Aarhus University Hospital, the Karen Elise Jensen Foundation and the Danish Cancer Society (grant number R73-A4284-13-S17). The study sponsors had no influence on the study design, collection, analysis and interpretation of the data and in the writing of the report. 
1 Brincker H. Sarcoid reactions and sarcoidosis in Hodgkin's disease and other malignant lymphomata. Br J Cancer 1972; 26: 120-123.

2 Ji J, Shu X, Li X, et al. Cancer risk in hospitalised sarcoidosis patients: a follow-up study in Sweden. Ann Oncol 2009; 20: 1121-1126.

3 Le Jeune I, Gribbin J, West J, et al. The incidence of cancer in patients with idiopathic pulmonary fibrosis and sarcoidosis in the UK. Respir Med 2007; 101: 2534-2540.

4 Askling J, Grunewald J, Eklund A, et al. Increased risk for cancer following sarcoidosis. Am J Respir Crit Care Med 1999; 160: 1668-1672.

5 Rømer FK, Hommelgaard P, Schou G. Sarcoidosis and cancer revisited: a long-term follow-up study of 555 Danish sarcoidosis patients. Eur Respir J 1998; 12: 906-912.

6 Seersholm N, Vestbo J, Viskum K. Risk of malignant neoplasms in patients with pulmonary sarcoidosis. Thorax 1997; 52: 892-894.

7 Spagnolo P, Luppi F, Roversi P, et al. Sarcoidosis: challenging diagnostic aspects of an old disease. Am J Med 2012; 125: 118-125.

8 Iannuzzi MC, Rybicki BA, Teirstein AS. Sarcoidosis. N Engl J Med 2007; 357: 2153-2165.

9 Sorensen HT, Mellemkjaer L, Nielsen GL, et al. Skin cancers and non-hodgkin lymphoma among users of systemic glucocorticoids: a population-based cohort study. J Natl Cancer Inst 2004; 96: 709-711.

10 Andersen TF, Madsen M, Jorgensen J, et al. The Danish National Hospital Register. A valuable source of data for modern health sciences. Dan Med Bull 1999; 46: 263-268.

11 Gjerstorff ML. The Danish Cancer Registry. Scand J Public Health 2011; 39: Suppl. 7, 42-45.

12 Boffetta P, Rabkin CS, Gridley G. A cohort study of cancer among sarcoidosis patients. Int J Cancer 2009; 124: 2697-2700.

13 Tana C, Giamberardino MA, Di Gioacchino M, et al. Immunopathogenesis of sarcoidosis and risk of malignancy: a lost truth? Int J Immunopathol Pharmacol 2013; 26: 305-313.

14 Landgren O, Engels EA, Pfeiffer RM, et al. Autoimmunity and susceptibility to Hodgkin lymphoma: a population-based case-control study in Scandinavia. J Natl Cancer Inst 2006; 98: 1321-1330.

15 Smedby KE, Hjalgrim $\mathrm{H}$, Askling J, et al. Autoimmune and chronic inflammatory disorders and risk of non-Hodgkin lymphoma by subtype. J Natl Cancer Inst 2006; 98: 51-60.

\section{An integrated MDR-TB management programme results in favourable outcomes in northern Taiwan}

\section{To the Editor:}

Multidrug-resistant tuberculosis (MDR-TB) is caused by Mycobacterium tuberculosis that is resistant to at least isoniazid (INH) and rifampicin (RIF). MDR-TB is difficult to treat and has become an obstacle to tuberculosis control programmes worldwide [1]. The global burden of MDR-TB has been increasing and the World Health Organization (WHO) estimated there were 450000 incident MDR-TB cases in 2012 [2]. Based on drug-resistance surveys, $3.6 \%$ of patients newly diagnosed and $20.2 \%$ of patients previously treated for tuberculosis had MDR-TB [2]. MDR-TB demands treatment with second-line drugs that have a limited sterilising capacity, and are less effective and more toxic than first-line drugs. Among the estimated $20 \%$ of the worldwide MDR-TB cases that were enrolled in treatment in 2010 , only $48 \%$ were successfully treated [2]. High mortality rates and loss to follow-up are threatening to destabilise global tuberculosis control.

In Taiwan, MDR-TB occurred in $1 \%$ of new tuberculosis cases and in $6 \%$ of re-treated tuberculosis cases [3]. In addition, laboratory-based analyses revealed that $10 \%$ of MDR-TB cases in Taiwan were extensively drug-resistant (XDR-TB) [4]. Faced with the challenge of the low treatment success rate of MDR-TB, a designated, government-organised and hospital-initiated programme led by experienced pulmonary specialists with diligent case managers, and cooperative and integrated medical groups, providing comprehensive and high-quality medical care for MDR-TB cases, was implemented by the Centers for 